\title{
Analysis of the Application Effect of Health Education in Skin Care
}

\author{
Weijie Qi* \\ Beauty Supreme Beauty Salon, Yuncheng 044000, China. E-mail: qiweij@163.com
}

\begin{abstract}
Objective: to analyze the effect of health education intervention in clinical nursing of patients with skin diseases. Methods: from January 2018 to March 2019, 122 patients with skin diseases were admitted to our hospital and randomly divided into two groups, with 61 cases in each group. The selected patients gave informed consent to this study. The control group was given routine dermatological care, and the observation group was given systematic health education. The treatment effect, mood score and quality of life score of the two groups were compared. Results: the treatment time, recurrence times and outpatient treatment times of the observation group were lower than those of the control group, the difference was statistically significant $(\mathrm{P}<0.05)$; the HAMA score and HAMD score of the observation group after intervention were lower than the control group, the difference was statistically significant $(\mathrm{P}<0.05)$. Conclusion: the implementation of health education for patients with skin diseases during the nursing process can effectively promote the recovery of the condition and reduce the number of treatments and recurrences. At the same time, it can improve the patients' bad mental mood and improve the quality of life of the patients.
\end{abstract}

Keywords: Health Education; Skin Care; Application Effect

\section{Introduction}

Implementing health education for patients in the care of skin diseases is the key to nursing. Effective and perfect health education is adopted to improve the awareness of skin disease patients and their families about the disease, thereby improving the quality of treatment and reducing the incidence of complications, and promote patients' mental health, etc. Common skin diseases are chronic urticaria, eczema and psoriasis. The condition of these diseases often recurs and the course is relatively long. Bring great pressure to the patient's body and mind. Therefore, how to actively improve the treatment effect of skin disease patients, reduce their physical and mental stress, and improve their quality of life is an important task in the treatment process of skin disease patients.

\section{Materials and methods}

\subsection{Information}

From January 2018 to March 2019, 122 skin disease patients were admitted to our hospital, and they were randomly divided into two groups with 61 cases in each. The selected patients gave informed consent to this study. In the control group, there were 32 males and 29 females; they were 19-66 years old, with an average of $(38.3 \pm 5.9)$ years old. In the observation group, there were 34 males and 27 females; they were 20-65 years old, with an average of $(38.5 \pm 7.2)$ years old. There was no statistically significant difference between the two groups of general

Copyright (C) 2020 Weijie Qi

doi: $10.18686 /$ jn.v9i4.187

This is an open-access article distributed under the terms of the Creative Commons Attribution Non-Commercial License

(http://creativecommons.org/licenses/by-nc/4.0/), which permits unrestricted non-commercial use, distribution, and reproduction in any medium, provided the original work is properly cited. 
information $(\mathrm{P}>0.05)$, and they were comparable.

\subsection{Method}

The control group performed routine dermatological care: (1) the ward was disinfected with an electronic sterilization lamp every day, the room was ventilated, and bed sheets and personal clothing were changed regularly to keep it clean and hygienic; (2) instruct patients to pay attention to personal hygiene, not to make nails too long and trim them in time to prevent scratching and damage to the skin; (3) regularly observe the patient's skin condition changes, whether there is any allergies, etc., and notify the doctor in time to deal with any adverse conditions; (4) pay more attention to diet. During treatment, patients are advised not to eat foods or drinks that can induce or aggravate skin diseases to avoid adverse conditions; (5) inform patients to avoid using drugs that have sensitized them, guide medication methods and medication precautions, and strictly follow the doctor's prescription for medication.

The observation group implemented systematic health education interventions: (1) cognitive intervention: according to the patient's cultural background and understanding ability, use easy-to-understand language to introduce to the patient the knowledge of skin diseases, the treatment effect and the whole treatment process. Let patients understand the significance and purpose of clinical treatment, and the importance of adhering to the doctor's advice, so that they can better cooperate with clinical treatment and enhance patients' treatment compliance; (2) medication guidance: guide the medication for the drugs used in the treatment process of the patient. Firstly, introduce the usage, dosage, time of medication, precautions during medication, adverse reaction symptoms and corresponding countermeasures to the patient to improve the patient's self-management ability. Strengthen inspections of elderly patients suffering from cardiovascular and cerebrovascular diseases to prevent adverse conditions; (3) self-care guidance: instruct patients to eat reasonably, quit smoking and alcohol, and prohibit eating irritating foods, foods that are too sweet, seafood foods, and foods that are prone to allergies. The daily diet is mainly light, eat more fresh fruits and vitamin-rich foods. Secondly, instruct patients to develop a regular and good work and rest time, ensure adequate sleep, pay attention to the combination of work and rest, appropriate physical exercises to enhance their own re- sistance, and maintain healthy living habits. Finally, instruct patients to master some daily self-care health care methods for the corresponding skin diseases of the patients, so as to improve the patients' self-care ability; (4) psychological counseling: to strengthen communication with patients, on the one hand to give patients verbal comfort and guidance, on the other hand, through communication with patients to grasp the root cause of patients' bad mood. On this basis, targeted emotional counseling interventions are given according to the causes of the patients' bad emotions, so that the bad psychological factors can be effectively alleviated. On this basis, the patients will be introduced to typical successful cases of treatment. When conditions permit, these successful patients can be invited to "show up" to help patients build confidence in the success of disease treatment, and at the same time allow their family members to participate in the psychological counseling of patients. Enable patients to receive clinical treatment with a good attitude; (5) identification of drug allergy. A comprehensive evaluation of patients with a history of drug allergies is carried out, and allergy drug reminders are established in a prominent position of the hospital bed to record and mark which drugs the patient has had allergic adverse reactions to, effectively ensuring the safety of medication during the treatment of patients.

\subsection{Efficacy evaluation}

Significantly effective: the patient's clinical symptoms have been significantly improved, and the number of repetitions of skin diseases is less or completely cured; improvement: the patient's clinical symptoms have improved, and the number of repetitions of skin diseases has decreased; invalid: the patient's clinical symptoms have not improved, the number of treatments and recurrence many times. Total effective rate $=$ apparent efficiency + improvement rate.

\subsection{Statistical processing}

Use SPSS 14.0 statistical software to process the data of this group. The count data is expressed in $\%$, and the $\mathrm{x}^{2}$ test is performed. When $\mathrm{P}<0.05$, it means that the difference in the comparison of this group is statistically significant.

\section{Results}




\subsection{Comparison of treatment effects between the two groups}

The treatment time of the observation group was shorter than that of the control group, the number of recurrences was less than that of the control group, and the number of outpatient treatments was less than that of the control group. The difference was statistically significant $(\mathrm{P}<0.05)$. See Table 1.

Table 1. Analysis of treatment effect of two groups of patients.

\begin{tabular}{|c|c|c|c|c|}
\hline Group & $\mathrm{n}$ & Treatment Time (d) & $\begin{array}{c}\text { Number of relapses } \\
\text { (times) }\end{array}$ & $\begin{array}{l}\text { Number of outpatient treat- } \\
\text { ments (times) }\end{array}$ \\
\hline Observation group & 61 & $6.48 \pm 1.09$ & $2.17 \pm 0.54$ & $2.53 \pm 0.71$ \\
\hline Comparison group & 61 & $10.55 \pm 1.78$ & $5.64 \pm 1.55$ & $5.87 \pm 1.82$ \\
\hline $\mathrm{t}$ & & 7.2572 & 6.4815 & 6.0525 \\
\hline $\mathrm{p}$ & & $<0.05$ & $<0.05$ & $<0.05$ \\
\hline
\end{tabular}

2.2 Comparison of the emotional state of the two groups of patients

The HAMA score and HAMD score before intervention between the two groups were not statistically different $(\mathrm{P}>0.05)$; the HAMA score and HAMD score of the observation group after intervention were lower than those of the control group, and the difference was statistically significant $(\mathrm{P}<0.05)$. See Table 2.

Table 2. Comparison of emotional state of the two groups of patients.

\begin{tabular}{lllllll}
\hline \multirow{2}{*}{ Group } & $\mathrm{n}$ & HAMA & & & HAMD & \\
\cline { 5 - 6 } & & Before intervention & After intervention & & Before intervention & After intervention \\
\hline Observation & 61 & $53.45 \pm 6.55$ & $3.044 \pm 6.03$ & & $47.25 \pm 6.39$ & $28.06 \pm 4.33$ \\
group & 61 & $52.46 \pm 6.11$ & $47.08 \pm 6.22$ & & $47.52 \pm 6.06$ & $37.41 \pm 6.35$ \\
Comparison & & 1.1644 & 7.2534 & & 1.0836 & 6.4228 \\
group & $>0.05$ & $<0.05$ & & $>0.05$ & $<0.05$ \\
$\mathrm{t}$ & & & & & \\
$\mathrm{p}$ & & & & & \\
\hline
\end{tabular}

\section{Discussion}

Health education refers to organized, planned and systematic education methods that can encourage patients to reduce or eliminate some risk factors that affect their own health through behaviors and lifestyles that are conducive to their own health, thereby improving and promoting themselves physical and mental health. The implementation of health education in the process can effectively promote the recovery of the disease and reduce the number of treatments and relapses. Skin disease is one of the diseases that seriously affect people's normal life and communication. Its incidence is relatively high, and it is a frequently-occurring disease, and even a few seriously endanger the lives of patients. Studies have pointed out that psychological and social factors play a very critical role in the treatment of diseases. Because most skin diseases are difficult to eradicate and are prone to relapse, they have a serious impact on the quality of life, and there is an inferiority complex. As the treatment of skin diseases is a long-term treatment process, it is essential to strengthen care and avoid the recurrence of the disease. This study proves that long-term treatment is required for skin disease patients in nursing skin disease patients. The long course of disease, coupled with the interference of various symptoms, causes the patient to suffer from the disease for a long time, which will adversely affect the physical and mental health of the patient. Therefore, strengthening health education interventions for patients with skin diseases, improving their disease awareness, and improving their cognitive behavior and emotional state are of great significance for improving the efficacy.

From the data in Table 2, it can be seen that the levels of anxiety and depression of patients who implemented health education interventions were significantly lower than those of patients in conventional care, and the difference was statistically significant $(\mathrm{P}<0.05)$. This 
shows that health education intervention can effectively improve the bad mental mood of patients with skin diseases. This is mainly due to health education interventions, from multiple angles, to take various measures to actively improve patients' cognitive attitudes towards diseases and improve their self-care and health care capabilities, so that patients can treat diseases more objectively and better self-care to eliminate bad emotions caused by lack of relevant health knowledge. The data in Table 1 shows that health education can significantly improve the treatment effect of patients with skin diseases, not only can shorten the recovery time of patients, but also significantly reduce the recurrence rate. This is mainly due to the fact that during the implementation of health education, the factors that are not conducive to disease recovery can be improved both physically and mentally. Through cognitive intervention, patients can have an objective understanding of the disease and the importance of treatment according to doctor's orders; and psychological care can help patients. The mentality is more optimistic, and more actively cooperate with the treatment, so as to achieve the purpose of improving the efficacy. The improvement of curative effect and emotional state are the fundamental factors that promote the improvement of patients' quality of life.

In summary, health education can change the behavior of patients, strengthen patients' awareness of the disease, and make them more proactive in cooperating with treatment, thereby reducing the occurrence and recurrence of complications, eliminating psychological pressure, and promoting early recovery of patients health education intervention can reduce the patient's treatment time, improve the treatment effect, and at the same time improve the patient's bad psychological mood and improve the patient's quality of life.

\section{References}

1. Peng Q. Influencing factors of poor medication compliance of patients with chronic skin diseases and the nursing improvement methods. International Medicine and Health Guidance News 2019; 25(15): 2611-2613.

2. Pan J, Xiao W. Analysis of psychological features of patients with dermatological disease and effect of nursing intervention. China Foreign Medical Treatment 2018; 37(16): 141-143.

3. Wan J, Cheng N. Observe the effect of nurse-patient communication skills training on improving the quality of dermatology care (in Chinese). Journal of Clinical Medical Literature (Electronic Edition) 2019; 6(52): 114-115.

4. Li H. Study on the effect of nurse-patient communication training in the management of dermatology nurses (in Chinese). China Health Industry 2019; 16(8): 28-29.

5. He X. Application effect of nurse-patient communication skills training in improving the quality of dermatology care (in Chinese). Journal of Medical Aesthetice and Cosmetology 2019; 28(8): 74.

6. $\mathrm{He} \mathrm{X}, \mathrm{Xu} \mathrm{K}, \mathrm{Chen} \mathrm{M}$, et al. Investigation on the current situation of knowledge and behaviors of topical skin medication in patients with chronic skin diseases (in Chinese). Academic Journal of Guangzhou Medical College 2019; 47(3): 176-178.

7. He L. Observe the effect of psychological care on the satisfaction of patients with skin diseases (in Chinese). Renowned Doctor 2020; (1): 187.

8. Zhao Y. The effect of soothing nursing methods on patients with skin diseases (in Chinese). Journal of Dermatology and Venereology 2018; 40(3): 449450. 